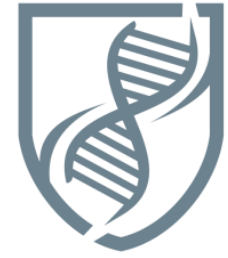

Journal of Bioscience and Applied Research

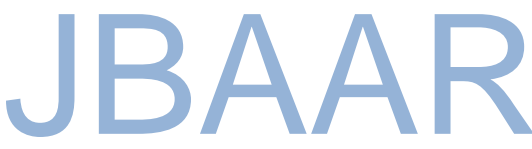

WWW.JBAAR.ORG

\title{
Ecophysiological and Histopathological Impacts of Organic Pollution on Two Freshwater Fish Species, Mansoura City, Egypt
}

Sherif H. Abdeen, Abeer E. Abdrabouh, Mohamed I. Mashaly, Ahmed E. Hagras,

$$
\text { Ali A. Al-halany* }
$$

Zoology Department, Faculty of Science, Mansoura University, Egypt

*Department of Biology, Faculty of Education, Hajjah University, Yemen

\section{DOI: $10.21608 /$ jbaar.2017.125860}

\begin{abstract}
Organic pollution is one of the environmental hazards, especially in aquatic ecosystems. This study was focusing on levels of total organic carbon (TOC\%) in the sediment of two freshwater habitats different in quality, as well as tissues including; muscles and gonads of two fish species inhabiting both sites during four seasons of one year of study. Ammar drain as a polluted site showed significantly higher levels of TOC\% in both sediment and fish tissues comparing to River Nile as a reference site. Data also illustrated that fish from the polluted site showed a remarkable decrease in blood indices; RBCs count, Hb content, and Hct\%, while WBCs count was mostly elevated, especially during autumn. Accompanying these results, antioxidant enzymes as superoxide dismutase (SOD) and catalase (CAT) were generally decreased during most seasons in fish samples from Ammar drain. Also, histopathological changes in muscles and gonads were observed, where to severe deteriorations in these tissues were more discriminating in fish species from Ammar drain compared to River Nile locality. From the obtained results, polluted water drains affect the general health and structure of their inhabiting fish species.
\end{abstract}

Keywords: Organic carbon, Fish, Antioxidants, Blood indices, Histopathology

\section{Introduction}

Although water is the most important subjected to danger as a result of increasing constituent of the ecosystem, it is highly human population, industrialization, and 
agricultural activities (Akan et al., 2012). These hazards are always discharged legally into drains which are closed systems without selfcleaning ability and therefore easily accumulate pollutants such as, heavy metals, organic matters, and microbial pollutants. These drains are usually complex and fragile ecosystems with bad aquatic life, especially for fish that unfortunately may be used for human consumption through the food chain (Abida et al.,2008). Some of these pollutants may be accumulated in the sediments, as well as benthic fauna or flora, the fundamental diet of different fish species, in a process known as bioaccumulation accompanied by food chain biomagnification (Lu et al.,2017). Organic matter plays a fundamental role in controlling the bioavailability and distribution of organic contaminants. The latter mainly comes from domestic wastes and agricultural activities that could seep into water bodies leading to an increase in microorganisms that consume water oxygen content and elevated biological oxygen demand (BOD),(Ben Ameur et al.,2012; Gwaski et al., 2013). Water or sediment content of organic substances can be expressed by detecting total organic carbon (TOC). The organic carbon content in river water was reported to be correlated with the size of a water region, climate, flora surrounding the watercourse, as well as the season of collecting the sample (Niemirycz et al., 2006).
Environmental biomonitoring programs introduced several tools used as biomarkers for the assessment of pollution hazards in aquatic ecosystems (Viarengo et al., 2007; Ben Ameur et al., 2012). Fish are sensitive bioindicators in assessing the health of aquatic ecosystems (Moharram et al., 2011; Karadag et al., 2014), where general health may be affected including; reproductive capacity and growth rate of different fish species (Moharram et al., 2011; Ben Ameur et al., 2012).

Several studies suggested that oxidative stress biomarkers are very important in the evaluation of fish health with different environmental stressors (Monterio et al.,2007; Pavlovic et al.,2010). Moreover, histological investigations can also represent early diagnostic tools by the identification of changes at the sub-organismal level (Adeogun et al., 2012). Thus, the present study highlighted a comparable evaluation of hematological, oxidative stress parameters and histopathological changes in the African catfish (Clarias gariepinus) and the white Nile tilapia (Oreochromis niloticus niloticus) as the most important edible fish inhabiting two different freshwater habitats at Dakahlia governorate, Egypt, depending on levels of TOC\% in sediment samples and different fish tissues (muscle, testis, ovary). 


\section{Materials and Methods}

\subsection{Sampling Areas}

Sediment and water samples were collected seasonally from two freshwater ecosystems that differed in quality, both at Dakahlia Governorate, Egypt. The first site (as a polluted environment) was Drain No.2, commonly known as Ammar drain that begins at Kafr Demerah Al-Gadeeda, located in the vicinity of Belquas city,50 km north Mansoura city and about $36 \mathrm{~km}$ from Gamasa city. The other site that acted as a reference site was a part of the Damietta branch of the River Nile located at Kafr Al-Tawilah, Talkha city (Figure 1).

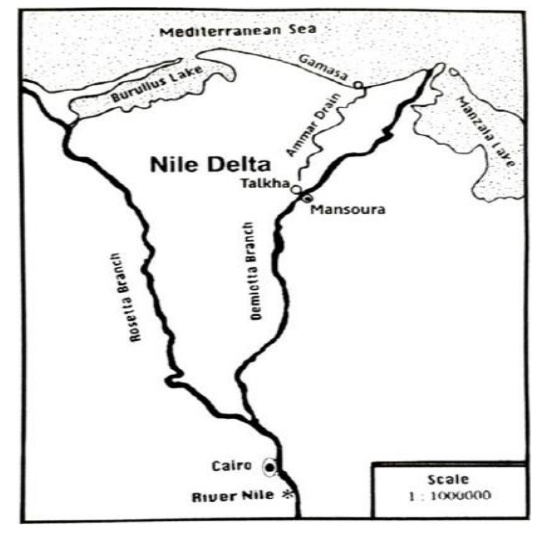

Figure 1. Map of the Nile Delta illustrating the Two Investigated sites

\subsection{Sediment Sampling}

During the period from March 2015 to February 2016 sediment samples were obtained monthly by using hand trowel help in collecting the top layer sediments, which were filtered from excess water and allowed to dry in the air, then crushed into fine granules in which total organic carbon (TOC) was detected using Winkler Black titration method described by (Hesse, 1971).

\subsection{Fish Sampling}

Several about (10-15) samples were collected seasonally from each of the studied fish species; Clarias gariepinus and Oreochromis niloticus niloticus from the two investigated sites during the study period. All fish were hunted by the same fisherman to prove the regular fishing method. Fish were transferred in containers filled with their water environment to the laboratory and then aerated in an aquarium using air pumps.

\subsection{Fish Preparation}

\subsubsection{Blood and Serum investigations}

From both investigated fish species; $C$. gariepinus and $O . n$. niloticus, blood samples were collected from the caudal vein. Each sample was divided into two portions; few drops on EDTA tube for the same-day assessment of hematological parameters; red blood cells (RBCs) count, hemoglobin $(\mathrm{Hb})$ content, hematocrit percentage (Hct\%), and white blood cells (WBCs) count, using the fully automatic hematological analyzer (Sysmex XE2100, Japan) according to Dacie and Lewis (2001). 
The rest of the obtained blood sample was collected in a centrifuge tube without anticoagulant, centrifuged at $3000 \mathrm{rpm}$ for 10 minutes. Clear serum samples were preserved at $-80^{\circ} \mathrm{C}$ till analysis for levels of superoxide dismutase (SOD) and catalase (CAT) using the method described by Candan and Tuzmen (2008).

\subsubsection{Tissue Investigations}

Following blood withdrawal, fish were dissected to obtain gonads (testes and ovaries), as well as a part of the dorsal muscle. Each tissue was divided into two portions; some tissues were lifted to dry overnight in an electric oven at $75^{\circ} \mathrm{C}$ until complete dryness for detection of TOC \% according to (Hesse,1971) as mentioned above, while other tissues were fixed in $10 \%$ formaldehyde for histological investigations using hematoxylin and eosin stain according to Alagappan et al.(2009).

\section{Statistical Analysis}

The present data were analyzed using the SPSS package (version 20), in which analysis of variance (one-way ANOVA) and Pearson's correlation coefficient tests was utilized to clarify the difference between the two studied localities and fish parameters, where $p$-value $\leq$ 0.05 was considered significant (Snedecor and Cochran,1982).

\section{Results}

The present work was conducted to evaluate the seasonal variation of TOC\% in sediment and fish tissues including; muscle and gonads (testis \& ovary) from two different freshwater localities; Ammar drain, as a polluted site, and River Nile at Altawela village, as a reference site.

\subsection{TOC \% in}

\section{sediment and tissues}

Table (1) showed seasonal fluctuations of TOC \% in sediment samples from the two investigated sites recording significant elevation in Ammar drain compared to River Nile site, through the four seasons of the study year, that was maximally observed during autumn. Moreover, TOC\% in male and female tissues (muscle, testis, ovary) either of $C$. gariepinus or O. n. niltocus was positively correlated with sediment TOC\% and maximally observed also during autumn, but non-significant for all tissue samples at both investigated sites during different seasons of the study year, Table 2 $(a, b)$.

\subsection{Hematological Parameters}

From Table $3(a, b)$, collected blood samples of the two investigated fish species from Ammar drain showed a general decrease in RBCs count, $\mathrm{Hb}$ content, and Hct\% compared to River 
Nile locality during the period of study that was observed during autumn. However, WBCs count showed a remarkable elevation in both species at the polluted site (Ammar drain).

\subsection{Oxidative stress enzymes}

Detected oxidative stress enzymes (SOD and CAT) levels in serum exhibited different patterns between both fish species at Ammar drain, where serum levels of SOD and CAT were remarkably decreased during all seasons, except spring for $C$. gariepinus, while $O$. n.niloticus showed the same reduction in both antioxidant enzymes during all seasons of investigation, except during spring and winter for SOD levels compared to the reference site as seen in Table $4(a, b)$.

Table1. Seasonal changes in total organic carbon (TOC \%) (Mean \pm SD) of sediment samples from the two investigated sites.

\begin{tabular}{|c||c|c|}
\hline Season & River Nile & Ammar Drain \\
\hline \multirow{2}{*}{ Spring } & 10.56 & 15.59 \\
& \pm 7.48 & \pm 9.27 \\
\cline { 2 - 3 } Summer & 13.16 & 24.10 \\
& \pm 5.43 & \pm 14.94 \\
\cline { 2 - 3 } Autumn & 4.46 & 29.90 \\
& \pm 4.58 & \pm 3.14 \\
\hline \multirow{2}{*}{ Winter } & 4.04 & 6.71 \\
& \pm 1.18 & \pm 1.66 \\
\hline
\end{tabular}


Table 2(a). Seasonal changes in the total organic carbon (TOC \%) (Mean \pm SD) in tissues of C. gariepinus from the two investigated sites.

\begin{tabular}{|c|c|c|c|c|c|c|c|c|}
\hline \multirow{3}{*}{ Season } & \multicolumn{4}{|c|}{ River Nile } & \multicolumn{4}{|c|}{ Ammar drain } \\
\hline & $\begin{array}{r}\mathrm{M} \\
\text { C. gar }\end{array}$ & $\begin{array}{l}\text { pinus } \\
\text { ping }\end{array}$ & $\begin{array}{r}\text { Fel } \\
\text { C. gar }\end{array}$ & $\begin{array}{l}\text { ale } \\
\text { pinus }\end{array}$ & $\begin{array}{r}\mathrm{M} \\
\text { C. gari }\end{array}$ & $\begin{array}{l}\mathrm{e} \\
\text { oinus }\end{array}$ & $\begin{array}{r}\text { Fen } \\
\text { C. garl }\end{array}$ & pinus \\
\hline & Muscle & Testis & Muscle & Ovary & Muscle & Testis & Muscle & Ovary \\
\hline Spring & $\begin{array}{r}24.56 \\
\pm 5.37\end{array}$ & $\begin{array}{l}20.60 \\
\pm 1.55\end{array}$ & $\begin{array}{r}22.79 \\
\pm 4.67\end{array}$ & $\begin{array}{r}22.38 \\
\pm 0.95\end{array}$ & $\begin{array}{r}28.34 \\
\pm 1.66\end{array}$ & $\begin{array}{r}28.17 \\
\pm 1.43\end{array}$ & $\begin{array}{r}28.19 \\
\pm 2.24\end{array}$ & $\begin{array}{l}27.41 \\
\pm 1.01\end{array}$ \\
\hline Summer & $\begin{array}{r}19.44 \\
\pm 9.06\end{array}$ & $\begin{array}{r}20.12 \\
\pm 6.64\end{array}$ & $\begin{array}{r}16.74 \\
\pm 9.20\end{array}$ & $\begin{array}{l}16.77 \\
\pm 6.04\end{array}$ & $\begin{array}{r}21.79 \\
\pm 6.39\end{array}$ & $\begin{array}{r}21.37 \\
\pm 2.34\end{array}$ & $\begin{array}{r}20.97 \\
\pm 4.65\end{array}$ & $\begin{array}{r}21.51 \\
\pm 1.52\end{array}$ \\
\hline Autumn & $\begin{array}{r}30.55 \\
\pm 6.20\end{array}$ & $\begin{array}{r}34.10 \\
\pm 6.08\end{array}$ & $\begin{array}{r}26.63 \\
\pm 9.24\end{array}$ & $\begin{array}{r}31.99 \\
+13.86\end{array}$ & $\begin{array}{r}38.21 \\
\pm 2.84\end{array}$ & $\begin{array}{r}38.41 \\
\pm 2.28\end{array}$ & $\begin{array}{r}29.91 \\
\pm 13.67\end{array}$ & $\begin{array}{r}34.28 \\
\pm 6.35\end{array}$ \\
\hline Winter & $\begin{array}{l}15.47 \\
\pm 3.30\end{array}$ & $\begin{array}{l}14.63 \\
\pm 1.95\end{array}$ & $\begin{array}{r}16.48 \\
\pm 2.78\end{array}$ & $\begin{array}{l}17.13 \\
\pm 3.03\end{array}$ & $\begin{array}{r}18.62 \\
\pm 0.93\end{array}$ & $\begin{array}{r}19.28 \\
\pm 0.06\end{array}$ & $\begin{array}{l}19.21 \\
\pm 0.10\end{array}$ & $\begin{array}{l}18.66 \\
\pm 0.96\end{array}$ \\
\hline
\end{tabular}


Table 2(b). Seasonal changes of the total organic carbon (TOC \%) (Mean \pm SD) in tissues of O. n. niloticus from the two investigated sites.

\begin{tabular}{|c|c|c|c|c|c|c|c|c|}
\hline \multirow{3}{*}{ Season } & \multicolumn{4}{|c|}{ River Nile } & \multicolumn{4}{|c|}{ Ammar Drain } \\
\hline & $\begin{array}{r}\text { M. n. } n \\
\text { o. }\end{array}$ & $\begin{array}{l}\mathbf{e} \\
\text { oticus }\end{array}$ & $\begin{array}{r}\text { Fen } \\
\text { o.n.n }\end{array}$ & $\begin{array}{l}\text { ale } \\
\text { oticus }\end{array}$ & $\begin{array}{r}\mathrm{M} \\
\text { o.n. n }\end{array}$ & $\begin{array}{l}\text { le } \\
\text { oticus }\end{array}$ & $\begin{array}{r}\text { Fen } \\
\text { o.n.n }\end{array}$ & $\begin{array}{l}\text { ale } \\
\text { oticus }\end{array}$ \\
\hline & Muscle & Testis & Muscle & Ovary & Muscle & Testis & Muscle & Ovary \\
\hline Spring & $\begin{array}{l}24.86 \\
\pm 4.85\end{array}$ & $\begin{array}{l}21.11 \\
\pm 1.17\end{array}$ & $\begin{array}{r}21.27 \\
\pm 1.02\end{array}$ & $\begin{array}{r}24.80 \\
\pm 4.82\end{array}$ & $\begin{array}{r}25.90 \\
\pm 4.01\end{array}$ & $\begin{array}{l}28.23 \\
\pm 2.03\end{array}$ & $\begin{array}{r}25.55 \\
\pm 1.67\end{array}$ & $\begin{array}{l}26.00 \\
\pm 1.46\end{array}$ \\
\hline Summer & $\begin{array}{l}19.76 \\
\pm 8.87\end{array}$ & $\begin{array}{r}17.88 \\
\pm 2.98\end{array}$ & $\begin{array}{r}13.36 \\
\pm 5.06\end{array}$ & $\begin{array}{r}17.69 \\
\pm 2.58\end{array}$ & $\begin{array}{r}25.84 \\
\pm 1.95\end{array}$ & $\begin{array}{l}20.64 \\
\pm 4.91\end{array}$ & $\begin{array}{l}21.64 \\
\pm 4.12\end{array}$ & $\begin{array}{l}21.76 \\
\pm 0.86\end{array}$ \\
\hline Autumn & $\begin{array}{l}34.50 \\
\pm 4.61\end{array}$ & $\begin{array}{r}28.36 \\
\pm 9.87\end{array}$ & $\begin{array}{r}34.06 \\
\pm 3.07\end{array}$ & $\begin{array}{l}31.49 \\
\pm 1.20\end{array}$ & $\begin{array}{r}39.99 \\
\pm 2.51\end{array}$ & $\begin{array}{l}37.70 \\
\pm 3.14\end{array}$ & $\begin{array}{l}37.67 \\
\pm 6.45\end{array}$ & $\begin{array}{l}34.68 \\
\pm 6.10\end{array}$ \\
\hline Winter & $\begin{array}{r}14.69 \\
\pm 5.35\end{array}$ & $\begin{array}{r}16.45 \\
\pm 2.73\end{array}$ & $\begin{array}{r}15.15 \\
\pm 3.47\end{array}$ & $\begin{array}{r}13.00 \\
\pm 3.94\end{array}$ & $\begin{array}{r}18.72 \\
\pm 0.76\end{array}$ & $\begin{array}{l}18.72 \\
\pm 0.76\end{array}$ & $\begin{array}{l}19.14 \\
\pm 0.05\end{array}$ & $\begin{array}{r}17.88 \\
\pm 1.13\end{array}$ \\
\hline
\end{tabular}


Table 3(a). Seasonal changes in the blood parameters (Mean \pm SD) of $C$. gariepinus from the two investigated sites.

\begin{tabular}{|c|c|c|c|c|c|c|c|c|}
\hline \multirow[b]{2}{*}{ Season } & \multicolumn{4}{|c|}{ River Nile } & \multicolumn{4}{|c|}{ Ammar Drain } \\
\hline & $\underset{\left(\times 10^{6} / \mu \mathrm{L}\right)}{\text { RBCs }}$ & $\begin{array}{c}\text { Hb } \\
\text { (g/dl) }\end{array}$ & $\begin{array}{l}\text { Het } \\
(\%)\end{array}$ & $\begin{array}{c}\text { WBCs } \\
\left(\times 10^{3} / \mu \mathrm{L}\right)\end{array}$ & $\begin{array}{c}\text { RBCs } \\
\left(\times 10^{6} / \mu \mathrm{L}\right)\end{array}$ & $\begin{array}{c}\mathbf{H b} \\
\text { (g/dl) }\end{array}$ & $\begin{array}{l}\text { Het } \\
(\%)\end{array}$ & $\begin{array}{c}\text { WBCs } \\
\left(\times 10^{3} / \mu \mathrm{L}\right)\end{array}$ \\
\hline Spring & $\begin{array}{c}2.21 \\
\pm 0.74\end{array}$ & $\begin{array}{c}9.93 \\
\pm 3.42\end{array}$ & $\begin{array}{l}34.37 \\
\pm 8.87\end{array}$ & $\begin{array}{l}207.13 \\
\pm 27.39\end{array}$ & $\begin{array}{c}2.06 \\
\pm 0.02\end{array}$ & $\begin{array}{c}9.73 \\
\pm \\
0.40\end{array}$ & $\begin{array}{l}28.47 \\
\pm 2.25\end{array}$ & $\begin{array}{l}209.43 \\
\pm 20.59\end{array}$ \\
\hline Summer & $\begin{array}{c}2.34 \\
\pm 0.28 \\
\end{array}$ & $\begin{array}{c}9.83 \\
\pm 1.79 \\
\end{array}$ & $\begin{array}{r}30.33 \\
\pm 5.15 \\
\end{array}$ & $\begin{array}{r}179.77 \\
\pm 16.54 \\
\end{array}$ & $\begin{array}{c}1.73 \\
\pm 0.48\end{array}$ & $\begin{array}{c}7.13 \\
\pm 2.14\end{array}$ & $\begin{array}{l}24.03 \\
\pm 6.38\end{array}$ & $\begin{array}{l}201.63 \\
\pm 13.68\end{array}$ \\
\hline Autumn & $\begin{array}{c}2.63 \\
\pm 0.14\end{array}$ & $\begin{array}{r}11.43 \\
\pm 0.46 \\
\end{array}$ & $\begin{array}{l}38.33 \\
\pm 4.47\end{array}$ & $\begin{array}{r}187.23 \\
\pm 25.99\end{array}$ & $\begin{array}{c}1.91 \\
\pm 0.82\end{array}$ & $\begin{array}{c}9.40 \\
\pm 1.75\end{array}$ & $\begin{array}{l}26.13 \\
\pm 7.52\end{array}$ & $\begin{array}{c}214.13 \\
\pm 2.30\end{array}$ \\
\hline Winter & $\begin{array}{c}2.87 \\
\pm 0.61\end{array}$ & $\begin{array}{r}17.33 \\
\pm 3.26\end{array}$ & $\begin{array}{l}35.28 \\
\pm 7.38\end{array}$ & $\begin{array}{r}161.23 \\
\pm 32.35\end{array}$ & $\begin{array}{c}2.16 \\
\pm 0.40\end{array}$ & $\begin{array}{c}9.50 \\
\pm 1.56\end{array}$ & $\begin{array}{l}26.93 \\
\pm 3.54\end{array}$ & $\begin{array}{l}204.00 \\
\pm 11.53\end{array}$ \\
\hline
\end{tabular}

RBCs: Red blood cells, Hb: Hemoglobin, Hct: hematocrit, WBCs: White blood cells

Table 3(b). Seasonal changes in the blood parameters (Mean \pm SD) of $O$. $n$. niloticus from the two investigated sites.

\begin{tabular}{|c|c|c|c|c|c|c|c|c|}
\hline \multirow[b]{2}{*}{ Season } & \multicolumn{4}{|c|}{ River Nile } & \multicolumn{4}{|c|}{ Ammar Drain } \\
\hline & $\begin{array}{c}\text { RBCs } \\
\left(\times 10^{6} / \mu \mathrm{L}\right)\end{array}$ & $\begin{array}{c}\text { Hb } \\
\text { (g/dl) }\end{array}$ & $\begin{array}{l}\text { Het } \\
(\%)\end{array}$ & $\begin{array}{c}\text { WBCs } \\
\left(\times 10^{3} / \mu \mathrm{L}\right)\end{array}$ & $\begin{array}{c}\text { RBCs } \\
\left(\times 10^{6} / \mu \mathrm{L}\right)\end{array}$ & $\begin{array}{c}\text { Hb } \\
\text { (g/dl) }\end{array}$ & $\begin{array}{l}\text { Hct } \\
(\%)\end{array}$ & $\begin{array}{c}\text { WBCs } \\
\left(\times 10^{3} / \mu \mathrm{L}\right)\end{array}$ \\
\hline Spring & $\begin{array}{c}1.61 \\
\pm 0.07\end{array}$ & $\begin{array}{c}9.87 \\
\pm 0.51\end{array}$ & $\begin{array}{l}27.40 \\
\pm 1.23\end{array}$ & $\begin{array}{r}191.00 \\
\pm 10.95\end{array}$ & $\begin{array}{c}1.60 \\
\pm 0.02\end{array}$ & $\begin{array}{c}8.07 \\
\pm 0.45\end{array}$ & $\begin{array}{l}25.87 \\
\pm 1.06\end{array}$ & $\begin{array}{l}210.57 \\
\pm 4.67\end{array}$ \\
\hline Summer & $\begin{array}{c}2.34 \\
\pm 0.15 \\
\end{array}$ & $\begin{array}{c}9.80 \\
\pm 0.10 \\
\end{array}$ & $\begin{array}{l}32.13 \\
\pm 2.23 \\
\end{array}$ & $\begin{array}{l}201.23 \\
\pm 6.05 \\
\end{array}$ & $\begin{array}{c}1.84 \\
\pm 0.45 \\
\end{array}$ & $\begin{array}{c}7.80 \\
\pm 2.86 \\
\end{array}$ & $\begin{array}{l}30.27 \\
\pm 6.70 \\
\end{array}$ & $\begin{array}{l}201.63 \\
\pm 13.68 \\
\end{array}$ \\
\hline Autumn & $\begin{array}{c}2.09 \\
\pm 0.62 \\
\end{array}$ & $\begin{array}{r}8.27 \\
\pm 1.29 \\
\end{array}$ & $\begin{array}{l}30.07 \\
\pm 4.39\end{array}$ & $\begin{array}{r}192.10 \\
\pm 12.44 \\
\end{array}$ & $\begin{array}{c}1.81 \\
\pm 0.30\end{array}$ & $\begin{array}{c}7.47 \\
\pm 1.19 \\
\end{array}$ & $\begin{array}{l}29.47 \\
\pm 5.35\end{array}$ & $\begin{array}{l}201.87 \\
\pm 20.80\end{array}$ \\
\hline Winter & $\begin{array}{c}1.66 \\
\pm 0.24 \\
\end{array}$ & $\begin{array}{l}11.43 \\
\pm 0.51 \\
\end{array}$ & $\begin{array}{l}26.11 \\
\pm 1.36 \\
\end{array}$ & $\begin{array}{l}126.27 \\
\pm 2.65\end{array}$ & $\begin{array}{r}1.46 \\
\pm 0.27 \\
\end{array}$ & $\begin{array}{c}8.33 \\
\pm 1.82 \\
\end{array}$ & $\begin{array}{l}24.67 \\
\pm 4.58 \\
\end{array}$ & $\begin{array}{r}199.33 \\
\pm 22.50 \\
\end{array}$ \\
\hline
\end{tabular}

RBCs: Red blood cells, Hb: Hemoglobin, Hct: hematocrit, WBCs: White blood cells 
Table 4 (a). Seasonal changes in the serum antioxidants (Mean \pm SD) of $C$. gariepinus from the two investigated sites.

\begin{tabular}{|c||c|c||c|c|}
\hline \multirow{2}{*}{ Season } & \multicolumn{2}{c|}{ River Nile } & \multicolumn{2}{c|}{ Ammar Drain } \\
\cline { 2 - 3 } & $\begin{array}{c}\text { SOD } \\
(\mathbf{u} / \mathbf{m l})\end{array}$ & $\begin{array}{c}\text { CAT } \\
(\mathbf{u} / \mathbf{l})\end{array}$ & $\begin{array}{c}\text { SOD } \\
(\mathbf{u} / \mathbf{m l})\end{array}$ & $\begin{array}{c}\text { CAT } \\
(\mathbf{u} / \mathbf{l})\end{array}$ \\
\hline \multirow{2}{*}{ Spring } & 137.50 & 422.67 & 175.00 & 869.10 \\
& \pm 57.28 & \pm 32.13 & \pm 78.06 & \pm 137.04 \\
\hline \multirow{2}{*}{ Summer } & 270.73 & 150.60 & 145.76 & 115.27 \\
& \pm 36.08 & \pm 33.90 & \pm 95.43 & \pm 55.33 \\
\hline \multirow{2}{*}{ Autumn } & 267.04 & 610.31 & 144.14 & 363.81 \\
& \pm 20.61 & \pm 77.56 & \pm 52.03 & \pm 13.92 \\
\hline \multirow{2}{*}{ Winter } & 341.50 & 148.80 & 245.72 & 109.10 \\
& \pm 14.50 & \pm 15.21 & \pm 25.93 & \pm 82.75 \\
\hline \hline
\end{tabular}

SOD: Superoxide dismutase, CAT: Catalase enzymes

Table 4(b). Seasonal changes in the serum antioxidants (Mean \pm SD) of $O$. $n$. niloticus from the two investigated sites.

\begin{tabular}{||c|c|c|c|c||}
\hline \multirow{2}{*}{ Season } & \multicolumn{2}{|c|}{ River Nile } & \multicolumn{2}{c|}{ Ammar Drain } \\
\cline { 2 - 5 } & $\begin{array}{c}\text { SOD } \\
(\mathbf{u} / \mathbf{m l})\end{array}$ & $\begin{array}{c}\text { CAT } \\
(\mathbf{u} / \mathbf{l})\end{array}$ & $\begin{array}{c}\text { SOD } \\
(\mathbf{u} / \mathbf{m l})\end{array}$ & $\begin{array}{c}\text { CAT } \\
(\mathbf{u} / \mathbf{l})\end{array}$ \\
\hline \multirow{2}{*}{ Spring } & 175.00 & 869.10 & 225.00 & 605.00 \\
& \pm 78.06 & \pm 137.04 & \pm 75.00 & \pm 180.90 \\
\hline \multirow{2}{*}{ Summer } & 249.93 & 750.13 & 145.77 & 472.43 \\
& \pm 62.45 & \pm 192.20 & \pm 36.14 & \pm 111.60 \\
\hline \multirow{2}{*}{ Autumn } & 246.08 & 917.16 & 126.73 & 669.31 \\
& \pm 34.18 & \pm 80.28 & \pm 35.20 & \pm 147.13 \\
\hline \multirow{2}{*}{ Winter } & 299.87 & 324.77 & 337.37 & 231.91 \\
& \pm 54.37 & \pm 391.15 & \pm 12.56 & \pm 125.36 \\
\hline \hline
\end{tabular}

SOD: Superoxide dismutase, CAT: Catalase enzymes

\subsection{Histopathological changes}

\subsubsection{Muscles}

Histological investigation in muscles of the two studied fish species collected from the River Nile showed polygonal muscle bundles formed of well-organized closely-packed units for $C$. gariepinus and $O$. n. niloticus (Figures 2 $\& 2 \backslash$ A, B) during winter and summer, respectively. However, at Ammar drain, marked deformation of muscle bundles represented by loosened and widely-spaced muscle fibers with clumps of inflammatory cells (granulomas) and encysted parasitic form was enveloped by loosened fibrous tissue, as shown in Figures $2 \& 2^{\backslash}(\mathrm{C}, \mathrm{D})$ for $C$. 
gariepinus and O.n. niloticus during winter and summer, respectively at both localities.

\subsubsection{Testes}

Fish species collected from the River Nile showed testicular lobules surrounded by interstitium showing a high density of centrally located spermatozoa and tubules were also surrounded by blood vessels, during winter and summer, Figures $3 \& 3^{\backslash}$ (A, B). In contrast, sections of testes from fish species obtained from Ammar drain Figures $3 \& 3^{\backslash}(\mathrm{C}, \mathrm{D})$ were characterized by distended testicular lobules that sometimes empty from spermatozoa, where the latter were seen scattered in the center of lobules, especially during winter, Figures $3 \& 3^{\backslash}(\mathrm{C})$ in both species.

\subsubsection{Ovaries}

The normal ovarian histology of both freshwater teleosts; African sharp tooth catfish
(C. gariepinus ) and Nile white tilapia (O.n. niloticus) was seen at the River Nile locality, where fully developed oocytes loaded with vitellioe cells were evident in addition to previtellogenic and postvitellogenic forms that occupied most of the cytoplasm, Figures $4 \& 4$ (A, B). Regarding fish samples from Ammar drain, Figures $4 \& 4^{\backslash}(\mathrm{C}, \mathrm{D})$ sections in ovaries showed remarkable degeneration and resorption of oocytes, vacuolation of a cytoplasmic matrix, disordered oogenesis, and collapse of germinal epithelium were evident, where this was more obvious during summer for both species, Figures $4 \& 4^{\backslash}$ (D).

N.B. For all investigated tissues, River Nile locality represented by A \& B during winter and summer, respectively. However, Ammar drain is demonstrated by Figures C \& D during winter and summer, respectively.
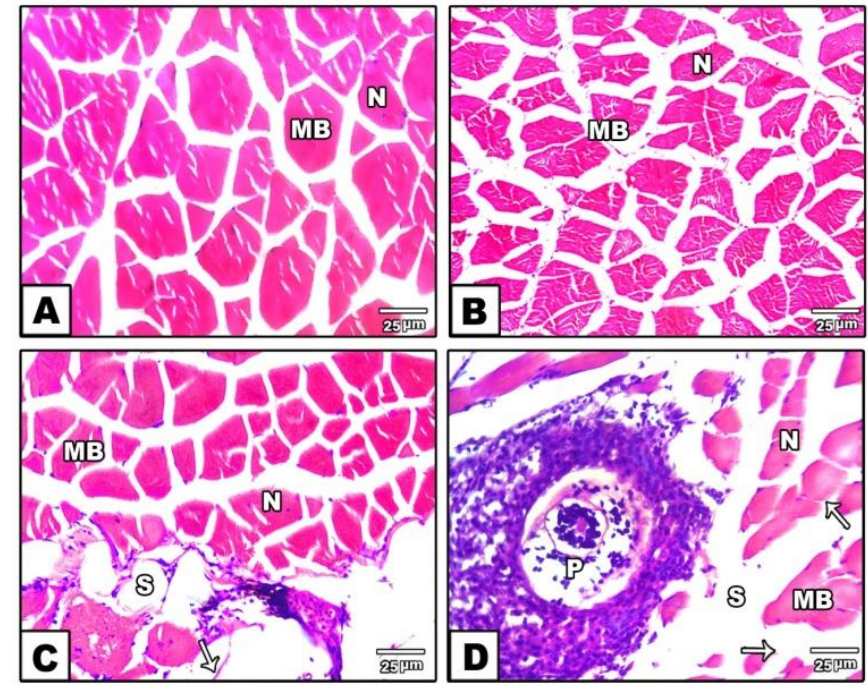

Figure (2). Photomicrograph showing the histological features of the muscle fibers of the African sharptooth catfish, Clarias gariepinus inhabiting the River Nile (A and B) and Ammar Drain (C and 
D). Scale bar $=25 \mu \mathrm{m}$. MB, muscle bundle; S, spaces between muscle bundles; $\mathrm{N}$, a nucleus of muscle bundle; $\mathrm{P}$, parasitic cyst; arrow, degenerated fibers.
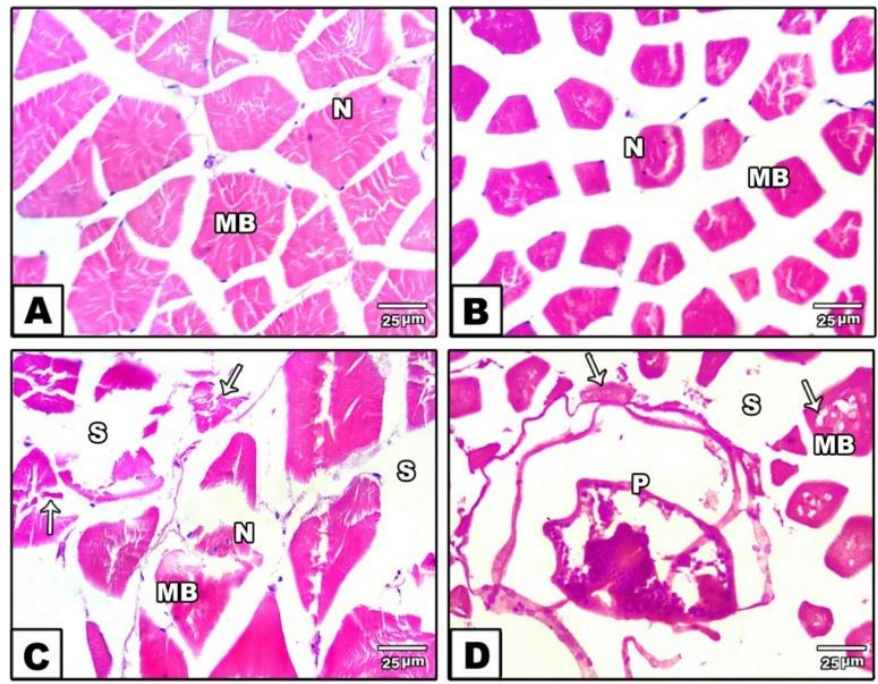

Figure ( $\left.2^{\prime}\right)$. Photomicrograph showing the histological features of the muscle fibers of the White Nile tilapia, Oreochromis niloticus niloticus dwelling the River Nile (A and B) and Ammar Drain (C and $\mathrm{D})$, during winter and summer, respectively. Scale bar $=25 \mu \mathrm{m}$. MB, a muscle bundle; S, spaces between muscle bundles; $\mathrm{N}$, a nucleus of the muscle bundle; $\mathrm{P}$, parasitic cyst; arrow, disintegrated fibers.

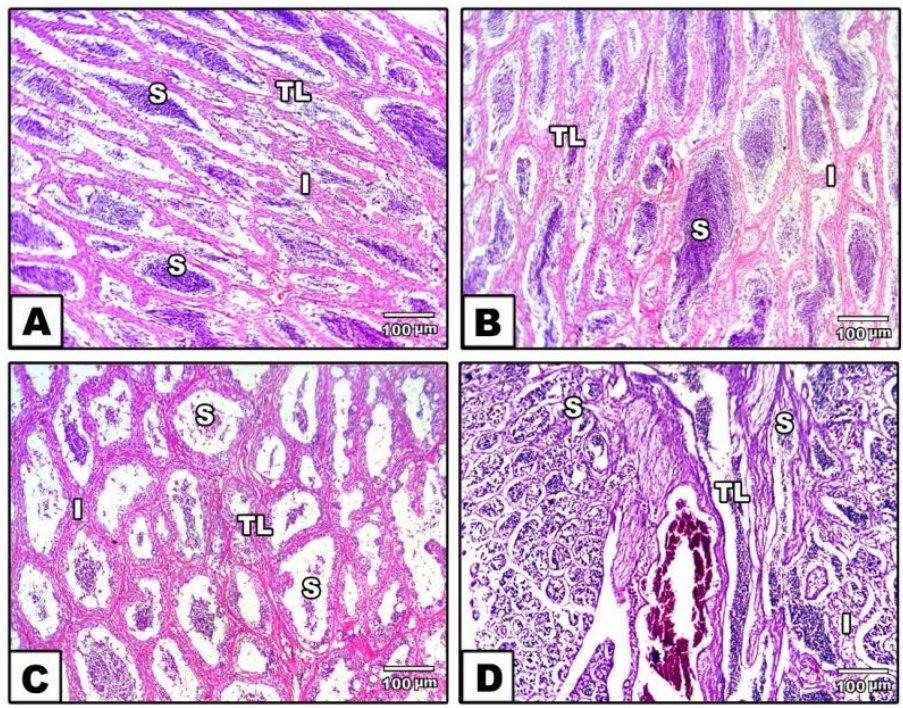

Figure (3). Photomicrograph showing the histological features of the testis of catfish, Clarias gariepinus dwelling in the River Nile during winter (A) and summer (B), and the histopathological features of some fish from Ammar Drain during winter (C) and summer (D). Scale bar $=100 \mu \mathrm{m}$. TL, testicular lobule; S, spermatozoa; I, interstitium. 

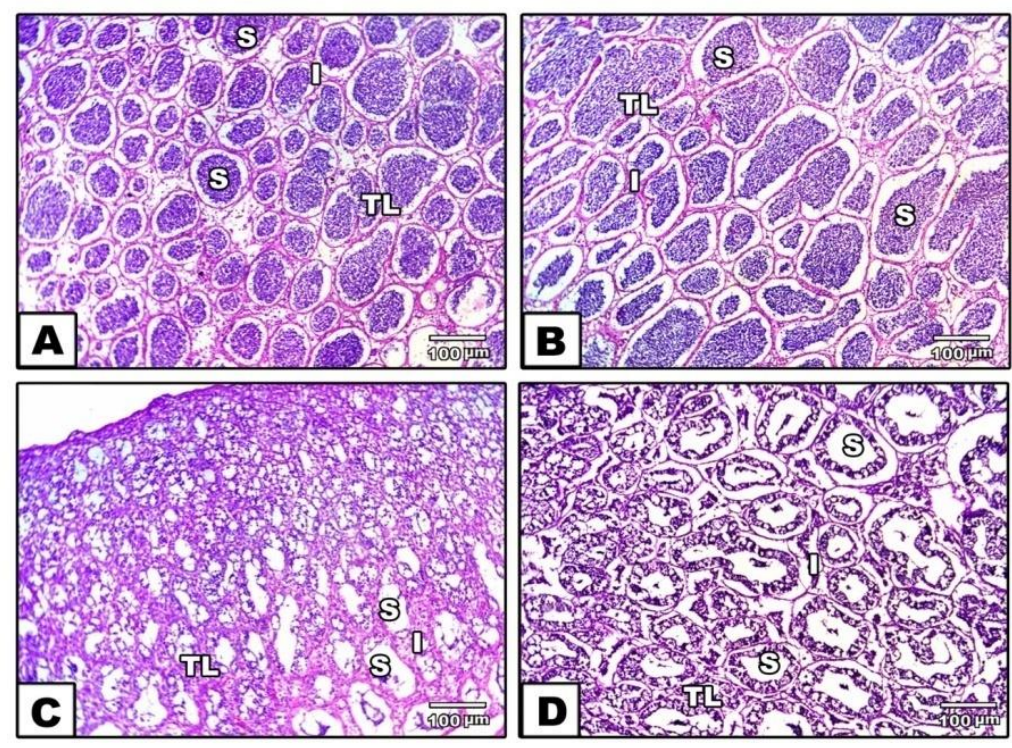

Figure $\left(3^{\prime}\right)$. Photomicrograph showing the histological features of the testis of Nile tilapia, Oreochromis niloticus niloticus from the River Nile during winter (A) and summer (B), and the histopathological features of the same fish from Ammar Drain during winter (C) and summer (D). Scale bar $=100 \mu \mathrm{m}$. TL, testicular lobule; S, spermatozoa; I, interstitium.
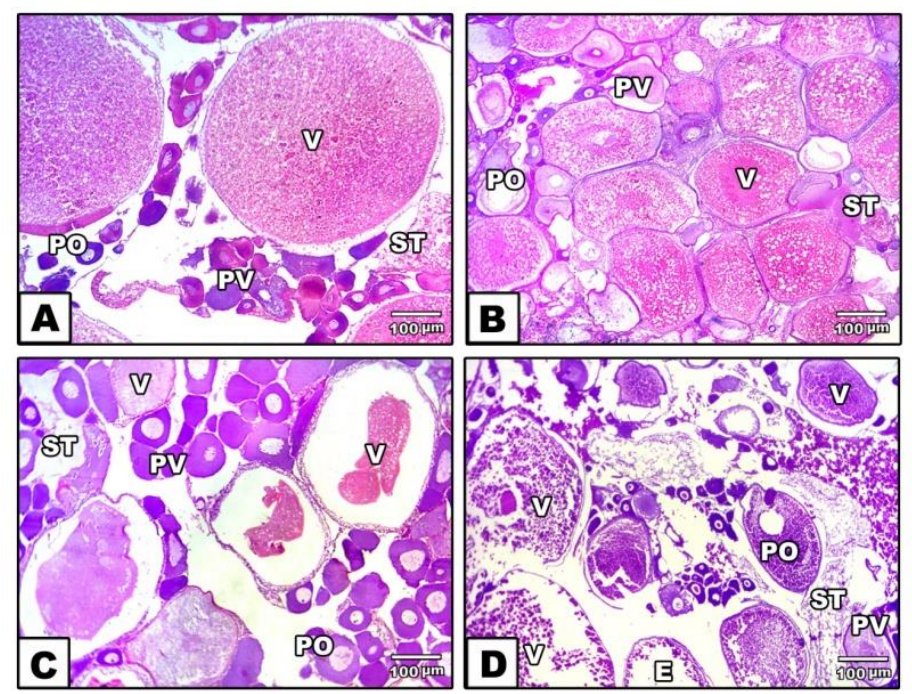

Figure (4). Photomicrograph showing the histological features of the ovary of catfish, Clarias gariepinus dwelling in the River Nile during winter (A) and summer (B), and the histopathological features of some fish from Ammar Drain during winter (C) and summer (D). Scale bar $=100 \mu \mathrm{m}$. E, empty follicle; PO, postvitellogenic stages; PV, previtellogenic stages; ST, stroma; V, vitellogenic stages. 

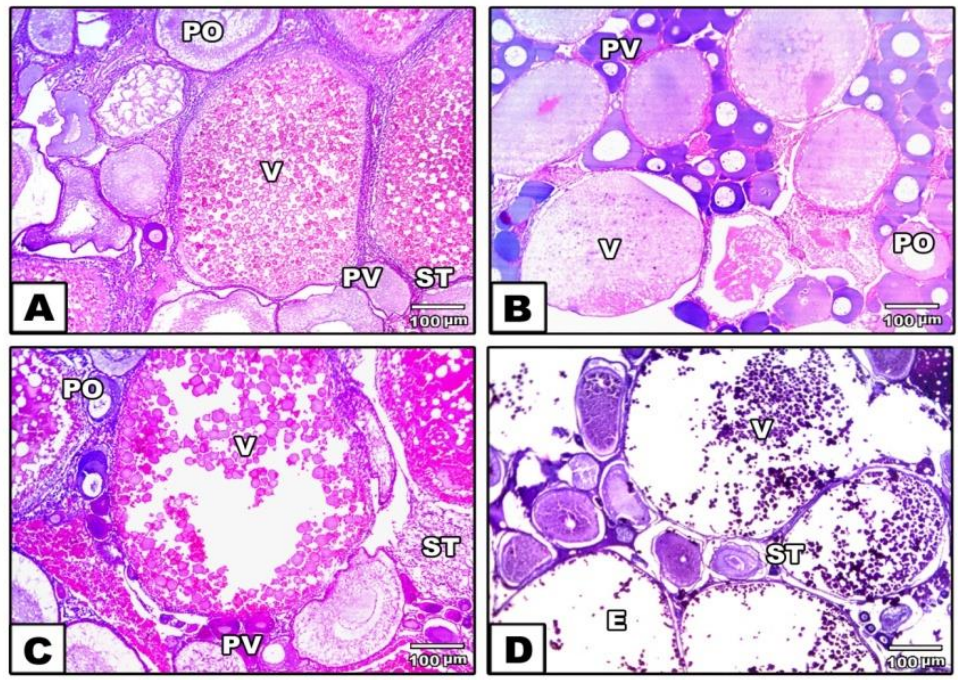

Figure (4'). Photomicrograph showing the histological features of the ovary of Nile tilapia, Oreochromis niloticus niloticus from the River Nile during winter (A) and summer (B), and the histopathological features of some fish from Ammar Drain during winter (C) and summer (D). Scale bar $=100 \mu \mathrm{m}$. E, empty follicle; PO, postvitellogenic stage; PV, previtellogenic stages; ST, stroma; V, vitellogenic stages.

\section{Discussion}

Human activities induce several environmental health hazards, that may affect hardly on aquatic organisms. Freshwater pollution by organic contaminants is one of these hazards. Total organic carbon (TOC) is a very important indicator in monitoring this problem (Abida et al.,2008; Akan et al.,2012). The present data showed that TOC\% in the sediment samples from Ammar drain was greater than that recorded of the River Nile locality. This could be attributed to the fact that Ammar drain received several agricultural and sewage wastes as the main source of organic carbon as recorded by (Donia,2005 and ElAmier et al.,2015). To follow this pollutant in the fish body, the present study was interested in studying TOC\% in fish tissues including; muscle, testis, and ovary of the two studied teleosts which were generally recorded higher levels in those inhabiting Ammar drain compared to the River Nile, confirming the higher levels detected in sediment samples. Accumulation of these pollutants may come through nutrition, where C.gariepinus and $O . n$. niloticus are known as omnivorous and herbivorous fish, respectively. In this concern, infaunal organisms and phytoplanktons, as first steps in the trophic levels were considered as important reservoirs for organic contaminants as reported by (Gunnarsson and Sköld,1999).

For more biomonitoring, some hematological, biochemical, and histopathological investigations were taken into consideration. Blood is a pathophysiological mirror of the health status of living organisms exposed to pollutants (Cazenave, 2005; 
Li, 2011). The potential oxygen-carrying capacity of the fish blood generally fluctuates with the biological life cycle, habitats, and physicochemical characters of the environment (Singh and Tandon,2009). The present work is complementary to a former paper published by El-Naggar et al. (2016), where a significant decrease in water oxygen content at Ammar drain was observed compared to River Nile locality accompanied by elevation of biological oxygen demand (BOD), as important indicators of increasing organic contamination in Ammar drain. In this concern, the present study showed depletion in RBCs count, $\mathrm{Hb}$ content, and $\mathrm{Hct} \%$ in blood samples of both fish species at Ammar drain compared to River Nile. Rifkind et al.(1980) explained that as these blood parameters are positively correlated with water oxygen content, where under oxygen depletion, the liver probably revives erythropoiesis to recompense the need for oxygen transportation to marginal tissues. Besides this, several studies revealed that the decrease in $\mathrm{RBCs}$ indices is due to RBCs lysis as a result of pollution by textile dyes on Prussian carp (AlSabti,2000), diazinon on Cyprinus carpio L. (Svoboda et al.,2001), cassava effluents on Oreochromis niloticus and Clarias gariepinus (Adekunle et al.,2007) and trace metals on Siganus rivulatus fish (Moharram et al.,2011).

In contrast, the present study also recorded remarkable elevation in WBCs in fish species collected from Ammar drain compared to those from the River Nile in a process known as leukocytosis, where leucocytes increased to protect the fish body against infection that may be increased with pollution as reported by Mazon et al. (2002) in case of Prochilodus scrofa exposed to copper.

On the other hand, water pollutants were considered as the main source of physiological disorders in fish (Adams et al.,2001; Viarengo et al.,2007). Water pollutants may lead to an oxidative stress condition, where reactive oxygen species (ROS) result in activation of antioxidant enzymes (Winston and Di Giulio,1991) or inhibited due to toxicity that may handicap the creation of reactive oxygen species (Cossu et al.,1997). Concerning antioxidant enzymes, the principal protection against undesirable ROS comprises SOD enzyme, which stimulates the change of ROS, such as superoxide anions to oxygen and hydrogen peroxide that converted by CAT into water (Ben Ameur et al.,2012). The enzyme response to pollutants usually displays an early elevation in an activity as a result of enzyme induction, followed by a decline in action as a result of improved catabolic pathways and/or inhibition by contaminants (Viarengo et $a l ., 2007)$ as seen in the present study, where a general decrease in serum SOD and CAT was recorded in both fish samples from Ammar drain compared to the River Nile site.

Reduction in SOD and CAT serum levels of both fish species at Ammar drain during most 
seasons of the study year, especially summer and autumn may be correlated with increased TOC\%, especially during autumn in sediment and tissue samples. This goes following several authors who connected between environmental pollutants and variations in levels of antioxidant enzymes (Adeogun et al., 2012; Ben Ameur et al., 2012; Karadag et al., 2014).

For more evaluation of fish health status, the present study was interested also in investigating histopathological changes in different fish' tissues including; muscles, testes, and ovaries. Muscles as the most edible part of the fish were widely affected in both investigated fish; C. gariepinus and O.n. niloticus from the polluted site (Ammar drain) compared to those from the reference site (River Nile). As shown in Figures 2(A-D) for $C$. gariepinus \& $2^{\backslash}$ (A-D) for O.n. niloticus during winter and summer at both localities, where degeneration, atrophy, and splitting of muscle fibers of both fish species collected from Ammar drain were observed comparing with River Nile fish species. This was in agreement with the results of (Patnaik et al., 2011) who explained that fish muscles are directly contacted with pollutants through water and sediments which may induce hyperactivity and excitability in fish species leading to lactic acid release that results in muscular fatigue.

Moreover, the histological structures of testes and ovaries of the two studied fish species; $C$. gariepinus, Figures 3 \& 4 (A- D) \& O.n. niloticus, Figures $3^{\backslash} \& 4^{\backslash}$ (A-D) during winter and summer showed detrimental development of gonads that were more obvious at Ammar drain compared to River Nile, due to the discharge of organic pollutants including, sewage and agricultural activities. Testes and ovaries are pairs of compact bodies. They are not directly contacted with pollutants, but they can be affected indirectly through their contact with blood that was highly influenced by pollution as mentioned above in the reduction of RBCs and $\mathrm{Hb}$ content. The affected fish blood goes to Sertoli cells that are responsible for the nutrition of the developing oocytes result in incomplete oogenesis. Parallel to that spermatogenesis is also affected by pollution as reported by (Pugazhvendan et al.,2009; Moharram et al., 2011). These degradations in gonads' histopathology supported our results (El-Naggar et al., 2016) that recorded significant low levels of sex steroid hormones and gonadosomatic index in both fish species collected from Ammar drain, where this may directly result due to disruptions of the pituitary-gonadal axis or gonadal damage with exposure to contaminants, as mentioned by (Ebrahimi and Taherianfard,2011).

\section{Conclusion}

Ammar drain was enriched in organic pollutants represented by the increased levels of organic carbon in water sediments in different seasons that subsequently accumulated in fish 
tissues through the food chain and affect the general health of inhabiting fish species represented by a reduction in blood indices and antioxidant enzymes accompanied by deteriorations in structures of different tissues in both investigated fish species compared to the River Nile, as a reference site.

\section{References}

Abida, B.; Harikrishna, S.; Irfanulla, K.; Ramaiah, M.; Veena, K. and Vinutha, K. (2008): "Analysis of Flouride Level in Water and Fish Species of Sankey, Bellandur, and Madi- vala Lakes of Bangalore. Rasayan," Journal of Chemistry, Vol. 1, No. 3, pp: 596601.

Adams S. M.; Giesy J. P.; Tremblay L. A. and Eason C. T. (2001): The use of biomarkers in ecological risk assessment: recommendations from the Christchurch conference on Biomarkers in Ecotoxicology. Biomarkers, 6: 1-6.

Adekunle I.M.; Arowolo T.A.; Omoniyi I.T. and Olibambi O.T. (2007): Risk assessment of nile tilapia (Oreochromis niloticus) and African mud catfish (Clarias gariepinus) exposed to cassava effluent. Chem. Ecol.,23:283-292.

Adeogun A. O. (2012): Impact of Industrial Effluent on Water Quality and Gill Pathology of Clarias gariepinus from Alaro Stream, Ibadan, Southwest, Nigeria. Europ. J. Scient. Res., 76 (1): 83-94.

Akan J. C.; Chellube M. T.; Chellube Z. M. and Abdulrahman F. I. (2012): Assessment of Pollutants in Water and Sediment Samples in Lake Chad, Baga, North-Eastern Nigeria. $J$. Environ.Protec., 3: 1428-1441.

\section{Alagappan K.M.; Deivasigamani B.;} Kumaran S. and Sakthivel M. (2009): Histopathological alterationsin Estuarine Catfish (Arius maculatus, Thunberg,1792) due to Aeronomas hydrophila infection. World J. Fish Marine Sci., 1(3):185-189.

Al-Sabti K. (2000): Chlorotriazine reactive azo red 120 textile dye induces micronuclei in fish. Ecotoxicol.Environ.Saf.,47: 149-155.

Ben Ameur. W.; Lapuente J. d.; EI Megdiche. Y.; Barhoumi B.; Trabelsi S.; Camps L. and et al., (2012): Oxidative stress, genotoxicity and histopathology biomarker responses in mullet (Mugil cephalus) and sea bass (Dicentrarchus labrax) liver from Bizerte Lagoon (Tunisia). Marine Poll. Bull., 64: 241251.

Candan N. and Tuzmen N. (2008): Very rapid quantification of malondialdehyde (MDA) in rat brain exposed to lead, aluminium and phenolic antioxidants by highperformance liquid chromatography-fluorescence detection. Neurotoxicol., 29: 708-713.

Cazenave J.D.A.; Wunderlin A. C. H. and Angeles-Bistoni M. D. (2005): Haematological parameters in a neotropical fish, Corydoras paleatus (Jenyns, 1842) (Pisces, Callichthyidae), captured from pristine and polluted water. Hydrobiol., 537: 25-33.

Cossu C.; Doyotte A.; Jacquin M. C.; Babut M.; Exinger A.; Vasseur P.; (1997): 
Glutathione reductase, selenium-dependent glutathione peroxidase, glutathione levels, and lipid peroxidation in freshwater bivalves, Unio tumidus, as biomarkers of aquatic contamination in field studies. Ecotoxicol. Environ. Saf.,Nov.,38(2):122-131.

Dacie J.V. and Lewis S.M. (2001): Practical Haematology, 9th edition. Churchill Livingstone, London.PP.633.

Donia N. (2005): Rosetta Branch Waste Load Allocation Model. 9th International Water Technology Conference, IWTC9, Sharm ElSheikh, 17-20 March, 277-288.

Ebrahimi M. and Taherianfard M. (2011): The effects of heavy metals exposure on reproductive systems of cyprinid fish from Kor River. 24 Iran. J. Fisher. Sci., 10(1): 13- 2011.

El-Amier Y. A.; Zahran M. A. and AlMamoori S. O. (2015) Environmental Changes along Damietta Branch of the River Nile, Egypt. J. Environ.Sci., Mansoura University, 44: 235-255.

El-Naggar A.M.; Abdeen S.H.; Hagras A.E.; Abdrabouh A.E.; Mashaly M.E. and AlHalani A.A. (2016): Impacts of fluctuations of physicochemical environmental parameters of aquatic ecosystems on somatic indices and sex hormones of the teleosts Clarias gariepinus and Oreochromis niloticus niloticus. J.Biosc.Appl.Res.,2(10):670-685.

Gunnarsson J. and Sköld M. (1999): Accumulation of polychlorinated biphe-nyls by the infaunal brittle stars Amphiura filiformis and A. chiajei:effects of eutrophication and selective feeding. Marine Ecol. Prog Ser. 186:173- 183.

Gwaski P. A.; Stephen S.; Hati N. P. and Ndahi V. O. (2013): Modeling Parameters of Oxygen Demand in the Aquatic Environment of Lake Chad for Depletion Estimation. ARPN J. Sci. Technol. Jan., 3(1): 116-123.

Hesse P. R. (1971): A Text Book of Soil Chemical Analysis. John Nurray Williams Clowes and sons Ltd. London, 324 pp.

Karadag H.; Firat O. and Firat O. (2014): Use of Oxidative Stress Biomarkers in Cyprinus carpio L. for the Evaluation of Water Pollution in Ataturk Dam Lake (Adiyaman, Turkey). Bull. Environ. Contam. Toxicol., 92:289-293.

Li Z. H.; Velisek J.; Grabic R.; Li P.; Kolarova J. and Randak T. (2011): Use of hematological and plasma biochemical parameters to assess the chronic effects of a fungicide propiconazole on a freshwater teleost. Chemosphere, 83: 572-578.

Lu Q.; Jürgens M. D.; Johnson A. C.; Graf C.; Sweetman A.; Crosse J. and Whitehead P. (2017): Persistent Organic Pollutants in sediment and fish in the River Thames Catchment (UK). Sci Total Environ., Jan. 15 (576):78-84.

Mazon A. F.; Monteiro E. A. S.; Pinheiro G. H. D.; Fernandes M. N. (2002): Hematological and physiological changes induced by Short-term exposure to copper in the freshwater fish, Prochildus scrofa. Brazil. 
J. Biol., São Carlos, 62 : 621-631.

Moharram S.G.; Wahbi O.M. and El-Gerisy Z.A.(2011):Effect of polluted water from the Egyptian Eastern Mediterranian Cost on reproductive,toxicological and hematological characteristics of Siganus rivulatus. Pakistan J.Biol.Sci.14(12):668-681.

Monterio D. A.; Almedia J. A.; Rantin F. T. and Kalinin A. L. (2007): Oxidative stress biomarkers in the freshwater Characid fish, Brycon cephalus, exposed to organophosphorus insecticides Follisuper 600 (methyl parathion). Comp. Biochem. Physiol. Toxicol. Pharmacol., 143 (2): 141-149.

\section{Niemirycz E.; Gozdek J. and Koszka-Maroń D.(2006): Variability of Organic Carbon in} Water and Sediments of the Odra River and Its Tributaries. Polish J. of Environ. Stud., 15 (4): 557-563.

Patnaik B.B.; Howrelia H.J.; Mathews T. and Selvanayagam M. (2011): Histopathology of gill, liver,muscle and brain of Cyprinus carpio communis L. exposed to sublethal concentration of lead and cadmium. Afric. J.Biotech., Sept.,10(57):12218-12223.

Pavlovic S. Z.; Borković Mitić S. S.; Radovanović T. B.; Perendija B. R.; Despotović S. G.; et al. (2010): Seasonal variations of the activity of antioxidants defense enzymes in the Red mullet (Mullus barbatus) from Adriatic sea. Mar. Drugs, 8 (3): 413-428.

Pugazhvendan S.R.; Narendiran N.J.; Kumaran R.G.; Kumaran S. and Alagappan
K.M. (2009): Effect of malathion toxicity in the freshwater fish Ophiocephalus punctatus-A histological and histochemical study. World $J$. Fish Marine Sci., 1(3):218-224.

Rifkind R. A.; Bank A.; Marks P. A.; Nossell H. L.; Ellison R. R. and Lindenbaum J. (1980): Fundamentals of Hematology, second ed., Yearbook Medical Publishers, Inc., Chicago.

Singh B .P and Tandon P.K. (2009): Effect of river water pollution on hematological parameters of fish, Wallago attu. Res. Environ. Life Sci., 2 (4): 211-214.

Snedecor J.P. and Cochran W. (1982):

Statistical methods. $7^{\mathrm{a}}$ ed. Aims, USA, The Iowa State University Press. 507 pp.

Svoboda M.; Luskova V.; Drastichova J. and Zlabek V. (2001): The effluent of diazinon on hematological indices of common carp (Cyprinus carpio L.). Acta Vet.Brno,70: 457465.

Viarengo A.; Lowe D.; Bolognesi C.; Fabbri E. and Koehler A. (2007): The Use of Biomarkers in Biomonitoring: a 2-tier Approach Assessing the Level of Pollutantinduced Stress Syndrome in Sentinel Organisms. Comp. Biochem. Physiol.,146 : 281-300.

Winston G. W. and Di Giulio R. T. (1991): Prooxidant and antioxidant mechanisms in aquatic organisms. Aqua. Toxicol., 19: 137161. 